\title{
Two dimensional fluidised bed reactor: Performance of a novel multi-vortex distributor
}

\author{
Hendrik Gideon Brink, Jean Saayman, Willie Nicol* \\ University of Pretoria, Department Chemical Engineering, University of Pretoria Main Campus, Corner \\ Lynwood \& Roper Street, Hatfield, Pretoria, 0002, South Africa
}

\begin{abstract}
The influence of the distributor configuration on interphase mass transfer, gas axial dispersion and bubble size was studied in a 2-D fluidised bed reactor for two types of distributor configurations; a novel multi-vortex (MV) distributor with tuyéres directed vertically and horizontally at different heights and a standard perforated plate distributor (baseline). The linear inlet velocity $\left(U_{0}\right)$ ranged between $0.1 \mathrm{~m} / \mathrm{s}$ and $0.35 \mathrm{~m} / \mathrm{s}$, with air as fluidising medium at ambient conditions. The ozone decomposition reaction over $\mathrm{Fe}_{2} \mathrm{O}_{3}$ impregnated $\mathrm{FCC}$ catalyst was used as an indirect measure of the performance of the FBR and it was found that the MV distributor causes a significant improvement ( $15 \%$ average) in the conversion efficiencies at all velocities tested. Bubble size measurements (using two separate techniques) indicated larger bubbles for the MV distributor, while the visual bubbling to turbulent transition boundary $\left(U_{c}\right)$ for the MV distributor was found to be lower than the baseline distributor. The interphase bubble-emulsion mass transfer was quantified using the model derived by Thompson et al. (1999) and was found to be $52 \%$ higher for the MV distributor than the baseline distributor. In addition the MV distributor exhibited near plug flow characteristics at velocities exceeding $U_{c}$, while this was not the case for the baseline distributor.
\end{abstract}

Keywords: Two dimensional fluidized bed reactor, multi-vortex distributor, ozone decomposition reaction, interphase mass transfer quantification 


\section{Introduction}

Low velocity catalytic gas-fluidisation (bubbling and turbulent regime) generally has lower overall reaction rates than predicted by the intrinsic kinetics of the catalyst. The phenomenon is primarily caused by interphase mass transfer limitations due to particle-fluid separation. This causes bubbles to form in the bed, which results in gas bypassing. Increasing the gas flow rate typically increases the bubble size in the bed which reduces the mass transfer rate between the bubbles and the solids; this can affect the overall reaction rate [1-6]. In addition to the interphase mass transfer, gas-backmixing in the emulsion phase can influence the reaction performance. Historically the main focus in rectifying this problem was therefore to reduce the bubble sizes in the fluidised bed reactor (FBR) [1,3,4,6-10].

Van Ommen et al. [1] and Klein van Willigen et al. [11] states that the disadvantages of FBRs can be overcome by manipulating the hydrodynamics, and subsequently decoupling some of the conflicting design objectives. Examples of these conflicting objectives are bubble size vs. high gas flow and conversion vs. backmixing. This can be done by either changing the manner in which the fluidising medium is introduced to the bed, or by manipulating the physical properties or state of the solid phase in the FBR. In both these cases either a change in the dynamics or a geometric change can be applied to the specific FBR property. A change in the dynamics of the fluidising medium would constitute dynamic changes to the flow into the FBR, while a geometric change would involve redistribution of gas by internals in the FBR or modification of the initial distribution system. Dynamic changes to the solid phase would entail a modification in the rate of change in the particle distribution in the bed while geometric changes would involve a change in the particle properties, e.g. the particle size distribution in the bed. Wong \& Baird [12] and Pence \& Beasley [13] altered the dynamics of the gas supply by applying continuous periodic variations and found a significant improvement in the quality of fluidisation which is a measure of the phase distribution in the bed. Kleijn van Willigen et al. [14] successfully reduced the bubble sizes in a FBR by manipulating the solid phase dynamics by applying an alternating electric field to the FBR. This polarised the particles and increased the interparticle forces in the bed. Sun \& Grace [15] and Beetstra et al. [16] studied the influence of particle size distribution, i.e. the geometry of the particles, on FBR performance and found that a higher fines content and a broader particle size distribution resulted in the greatest improvement in performance and reduction in bubble sizes in the FBR. Geometric manipulation of the gas supply and flow patterns in the FBR has been studied extensively in literature. This method includes the installation of internals in the bed and the design of the distribution system. Internals have been proven successful in breaking up bubbles [17], decreasing backmixing by dividing the FBR into compartments [18] and by shedding the wake from the bubbles as they pass the internals (specifically wire mesh internals) [19]. The design of the distribution system to the FBR is considered crucial to the quality of fluidisation as well as conversion of reactants in the distributor section of the bed [20] as it has been proven that as much as $50 \%$ conversion can take place in the shallow section less than $10 \mathrm{~cm}$ above the distributor [21]. It implies that the distributor can effectively manipulate the gas flow 
in the bed and this concept has been be used successfully in various studies to manipulate FBR hydrodynamics.

Sobrino et al. [22] tested the performance of bubble cap distributors compared to a perforated plate distributor and found that the perforated plate distributor induced earlier onset of turbulent behaviour in the FBR due to jetting from the plate. This is preferred since the most rigorous gas mixing was observed on transition to the turbulent fluidization regime [23-25]. Kleijn van Willigen et al. [11] tested the performance of a secondary fractal injection distributor and found a decrease in the bubble size with an accompanying decrease in the total number of bubbles. Yan et al. [26] found that the axial hold-up and radial distribution of solids were more uniform for a multi-tube distributor when compared to a multi-orifice distributor.

Ouyang \& Levenspiel [27], Chyang et al. [7,9,28], Sreenivasan \& Raghavan [8] and Wormsdecker et al. [10] compared the influence of horizontal injection of gas to induce a swirling bed FBR to the normal axial injection distributor. They found that the deficiency of the bubbling regime can be addressed by increasing the horizontal momentum in the bed. The gas from conventional FBR distributors (porous plate, perforated plate and multi- vertical nozzle) possesses only axial momentum which causes the gas at any point in the FBR to possess an axial component which significantly exceeds the radial or tangential components. The axial component of the gas velocity is responsible for the fluidisation of the particles in the bed, while the horizontal component is responsible for horizontal momentum being transferred to the bed. A deficiency in horizontal velocity components reduces the fluid movement and therefore the mixing in the FBR. Injecting gas at an angle of less than $90^{\circ}$ to the horizontal increases the horizontal component of the gas flow which results in better solids distribution and a subsequent increase in mass transfer. The reduction of the horizontal momentum higher up the bed can be counteracted by further decreasing the injection angle of gas to the FBR. It can therefore be deduced that the theoretical maximum horizontal momentum would be transferred to the FBR at an injection angle parallel to the distributor. Furthermore, Chyang et al. [9,28] found that the lateral dispersion and lateral mixing are significantly improved by using a tube and horizontal nozzle distributor, which results in an accompanying reduction of the dead zones in the FBR. Applying a radial force to the particles in the FBR by injecting gas at multiple points along the outer wall of the FBR was tested in a rotating FBR by De Wilde and De Broqueville [29]. The Torbed reactor induces the same radial motion by injecting the gas from the distributor at an angle [30]. This gas distribution design induced rotation of the particles and therefore increased the tangential and centrifugal forces on the particles.

There are numerous methods to quantify the contribution of a novel mixing scheme in a FBR. One approach is to quantify changes in specific hydrodynamic parameters like bubble size, bubble velocity, gas hold-up etc. The measured changes (improvements) can then be used to speculate on its contribution to the overall performance of a FBR. Alternatively, one can start from an overall performance measurement like the conversion of a catalysed chemical reaction in the bed. For atmospheric cold-flow columns the ozone decomposition reaction has proven itself as an ideally suited reaction in this regard [31]. The drawback of the overall performance measurement is that the underlying reason for the change (improvement) might not be clear. Lack of fundamental understanding will subsequently result in uncertainty in scale-up of the novel mixing scheme. Reactor 
models in conjunction with literature correlations can be used to investigate possible reasons, but the uniqueness of the specific system is not considered by the models and correlations might lead to an incorrect conclusion. It is therefore preferable to join the abovementioned methods. The analysis of the results from the overall performance measurement is then based on additional hydrodynamic measurements performed simultaneously in the same bed.

The novel multi vortex (MV) distributor tested in this study is based on the concept of increased cross-axial (horizontal) momentum. By introducing the fluidising medium parallel to the distributor at different injection heights the horizontal components of the gas velocity were increased. The MV distributor is tested against a baseline distributor (perforated plate) with a comparable open area ratio and orifice velocities. The performance of the MV distributor was determined by fitting the Thompson et al. $[32,33]$ model to the experimentally determined conversions for both distributors and analysing the fitted parameter values for physical significance. Additionally the bubble sizes were determined using a photographic method as well as a mathematical technique in which the pressure fluctuations in the bed were decomposed and analysed [34]. The bubble properties were incorporated into the fitted model. The experimental procedure was performed in a pseudo-2D FBR with FCC catalyst, impregnated with $\mathrm{Fe}_{2} \mathrm{O}_{3}$ for the ozone decomposition reaction. The experiments were done at ambient conditions for superficial velocities $\left(U_{0}\right)$ ranging from $0.1 \mathrm{~m} / \mathrm{s}$ to $0.35 \mathrm{~m} / \mathrm{s}$, which included the bubble-turbulent transition boundary for the system.

\section{Materials and Methods}

\section{1 $\quad$ Fluidised bed Reactor}

The reactor used for the experimental study was a Plexiglas pseudo two dimensional (pseudo-2D) FBR with thickness $25 \mathrm{~mm}$, width $400 \mathrm{~mm}$ and height $4.5 \mathrm{~m}$. A volute primary cyclone, to handle the high solids loading at the upper gas velocities, and a tangential secondary cyclone were used. Excess fines that bypassed the secondary cyclone were captured in filter bags connected after the secondary cyclone.

Saayman [35] includes the complete engineering drawings for the reactor. Figure 1 shows the piping and instrumentation for the experimental setup. The volumetric flow of reactor feed gas was measured using a vortex flow meter with a linear velocity range of between $0.1 \mathrm{~m} / \mathrm{s}$ and $0.6 \mathrm{~m} / \mathrm{s}$. The pressure across the distributor and the cyclones were measured using Rosemount Analytical differential pressure meters. Two high frequency pressure transmitters (Wika S-10, Range 0-1.6 barg, and maximum measurement frequency of $1000 \mathrm{~Hz}$ ) were inserted on the upper surface of the distributor and $0.3 \mathrm{~m}$ above the distributor. Dehumidified compressed air at $15^{\circ} \mathrm{C}$ was used as gas supply, the relative humidity $(\mathrm{RH})$ of the gas varied between $37 \%$ and $41 \%$. The air was dozed with ozone (generated in anEcoTec MZV1000) in order to have an inlet ozone concentration varying between 20 ppm and 100 ppm, such low concentrations allow for negligible heat generation. Pure oxygen was used as feed to the ozone generator to reduce $\mathrm{NO}_{\mathrm{x}}$ formation.

Initially $3.75 \mathrm{~kg}$ of catalyst was loaded to the reactor. The amount of inactive catalyst in the dipleg (no ozone feed to dipleg) was determined to be $0.75 \mathrm{~kg}$. This was done by shutting down the gas at various superficial velocities and measuring the 
collected height in the dipleg. This mass fraction remained more or less constant at all superficial velocities employed. The packed bed height was in the order of 400 $\mathrm{mm}$.

The $U_{0}$ was adjusted to the desired superficial velocity, between $0.1 \mathrm{~m} / \mathrm{s}-0.35 \mathrm{~m} / \mathrm{s}$, while the ozone inlet concentration was kept in the range $20 \mathrm{ppm}-100 \mathrm{ppm}$. The inlet ozone concentration was logged for $10 \mathrm{~min}$ to account for variations in the ozone concentration after which the analyser feed was changed to the outlet ozone concentration which was logged for $10 \mathrm{~min}$. The ozone inlet and outlet concentrations were determined by sampling the air in the plenum chamber and at a height of $4.1 \mathrm{~m}$ above the distributor. A UV-106 ozone analyser from 2B Technologies Inc. were used for the analysis at a wavelength of $254 \mathrm{~nm}$.

Data acquisition was done using National Instruments USB-6008 analogue signal data loggers connected to a PC. The readings from the ozone analyser were measured at a rate of $5 \mathrm{~Hz}$, the velocity measurements were logged at a rate of $20 \mathrm{~Hz}$ and the pressure fluctuations at a rate of $200 \mathrm{~Hz}$.

Mean conversions and variations were calculated with the following equations:

$$
\begin{gathered}
x=\left[\operatorname{mean}\left(C_{\text {ozone,IN }}\right)-\operatorname{mean}\left(C_{\text {ozone,OUT }}\right)\right] / \operatorname{mean}\left(C_{\text {ozone }, I N}\right) \\
\sigma_{x}^{2}=\left(\sigma_{\text {ozone }, I N}^{2}-\sigma_{\text {ozone,OUT }}^{2}\right) / \sigma_{\text {ozone,IN }}^{2}
\end{gathered}
$$

The distributors used in the experimental setup were:

- A triangular pitch perforated plate distributor with $35 \times 2 \mathrm{~mm}$ holes, with a porous cloth between the plenum chamber and the distributor, to prevent weepage. The cloth also increased the pressure drop over the distributor ( $75 \%$ to $150 \%$ of the pressure drop over the bed) and therefore improved the gas distribution over the distributor [36].

- A multi-vortex (MV) distributor consisting of $38 \times 1 / 16$ " OD tubes, with rectangular pitch, ejecting gas either horizontally or vertically in strategic directions to induce a gas flow pattern as shown in Figure 2. The Figure also contains a photograph of the MV distributor used in the experimental study. Glass wool was inserted into the nozzles to prevent weepage and increase the pressure drop for improved distribution $(150 \%$ to $760 \%$ of the pressure drop over the bed) [36].

The MV distributor was designed to improve the quality of fluidisation in the FBR by inducing localized vortices around five distinct sets of nozzles. The proposed vortices would theoretically increase the horizontal momentum at the surface of the distributor. This would in turn increase the lateral dispersion and mass transfer in the bed and decrease the formation of dead zones on the surface of the distributor [7-9].

The conduit sizing for the MV distributor was chosen to have nearly equal open area and gas injection velocities for both the baseline and MV distributors. The calculated ratio of superficial velocity to orifice velocities as well as open areas are shown in Table 1, from which can be seen that the open areas and injection velocity ratios for the baseline and MV distributor differ by approximately $7 \%$. It is assumed this difference is negligible. 


\subsection{Catalyst Preparation and Ozone Decomposition}

A small test reactor (50 $\mathrm{mm}$ height $\times 16.4 \mathrm{~mm}$ inner diameter) is connected in parallel to the FBR reactor (see Figure 1). It consists of a packed bed loaded with approximately $10 \mathrm{~g}$ of catalyst, sampled from the FBR using a tap at the bottom of the bed. The samples were collected whilst the FBR was in operation to monitor the catalyst activity at different times during the experiment. The amount of catalyst sampled was negligible relative to the bed inventory. Axial dispersion in this reactor was estimated to be negligible [37] and accordingly a plug flow model was used to determine the kinetics.

The catalyst in this study was produced by adding commercial FCC catalyst (support particle) to a mixture of $10 \%$ (wt) Ferric Nitrate solution. After stirring for one hour the mixture was dried overnight and then calcinated at $450{ }^{\circ} \mathrm{C}$ for approximately 1.4 hours, during which the $\mathrm{NO}_{2}$ gasses were released from the mixture [35]. According to literature [15,38-43], the ozone decomposition reaction is first order with respect to the ozone concentration and this was confirmed in the kinetic experiments given in Figure 3. The deactivation profile for a single catalyst batch exposed to ambient conditions is given in Figure 4. The initial drop in activity is most likely due to desorption of water from the catalyst, since the activity plateau (reached after two hours) could be obtained without the use of ozone. The activity plateau exhibited a fluctuating behaviour ( $k_{r}$ "'varies between 1.3 and 1.6 in Figure 4) most probably caused by fluctuations in $\mathrm{RH}$ of the compressed air. Spot checks were performed every four hours during fluidization experiments (kinetic testing of online catalyst samples) in order to confirm that $k_{r}$ "' remains within the established plateau span (or range), while the average of the spot check values was used in estimating the conversion efficiencies of an experimental run.

The catalyst particle size distributions were determined using a Melvern Mastersizer 2000 , the particle size distributions were determined once for every experimental run (the time the catalyst particles were not exposed to atmospheric conditions). The measured particle size distributions for both the distributor configurations remained relatively constant. The Sauter mean diameter was measured as $87 \mu \mathrm{m}$ and $84 \mu \mathrm{m}$ and varied by $8.3 \%$ and $4.6 \%$ over the time span of the experiments for the baseline distributor and MV distributor respectively. The respective standard deviations of the particle size distributions varied by $4.8 \%$ and $5.9 \%$.

\subsection{Additional measurements}

Most bubbles spanned the cross section of the pseudo-2D FBR making visible bubble size measurements possible. A12.1 megapixel digital camera (Sony Cyber shot DSCW230 12.1MP) was used, with a frame rate of $30 \mathrm{fps}$. A standard $300 \mathrm{~mm}$ ruler, marked at $1 \mathrm{~mm}$ intervals, was fixed to the column as a reference. Data analyses were done visually and logged in Microsoft $®$ Excel 2007. The column was filmed for a period of $30 \mathrm{~s}$, with the camera mounted at the ruler height. The linear velocities in both distributor configurations ranged from $0.1 \mathrm{~m} / \mathrm{s}$ to $0.35 \mathrm{~m} / \mathrm{s}$ in intervals of $0.05 \mathrm{~m} / \mathrm{s}$. Figure 5 shows an example of a frame-by-frame analysis done of the bubble sizes, in this case at a gas superficial velocity of $0.2 \mathrm{~m} / \mathrm{s}$.

A pressure fluctuation decomposition technique described by Van der Schaaf et al. [34] was employed to determine the relative bubble sizes in the column at a height of 
$300 \mathrm{~mm}$ above the distributor plate. The technique compares the coherent and incoherent pressure fluctuations in the bed, from which the comparative bubble sizes can be determined.

\section{Results and Discussions}

All reaction measurements are reported in terms of conversion efficiency, defined as the experimentally measured conversion divided by the maximum possible conversion the reactor could achieve (ideal plug flow reactor) at the given superficial velocity. It enables comparison between runs of different activities and is useful to visualise the reactor performance. Conversion efficiencies between the ideal value of one and the CSTR limit can be attributed to interfacial mass transfer as well axial dispersion effects, while efficiencies below the CSTR limit indicates a non-particle related interfacial mass transfer effect. The experimental results for the perforated plate distributor (baseline) as well as the newly designed distributor are shown in Figure 6. A total of four and six experimental runs (the time the catalyst particles were not exposed to atmospheric conditions) were completed with the baseline and MV distributor respectively. The error bars show one standard deviation from the average measured conversion, taken over the experimentally determined ozone measurements. As can be seen in Figure 6, there seems to be quite extensive scatter in the measured conversion efficiency, yet a prominent trend is exhibited in both cases. A very definite dip in the conversion efficiency takes place from $0.1 \mathrm{~m} / \mathrm{s}$ to approximately $0.3 \mathrm{~m} / \mathrm{s}$. This corresponds well to the measured results from Saayman [35]. To refine the results, i.e. filter the scatter to a more manageable trend, a moving average over 5 consecutive data point were applied to the data, the filtered data can be seen in Figure $7 a$.

The data shows a definite increase in the conversion efficiency when using the MV distributor as compared to the baseline distributor. Figure $7 \mathrm{~b}$ shows the percentage increase of the conversion efficiency for the new distributor. This increase varies between $0 \%$ and $20 \%$, with a mean improvement of $14.7 \%$ and an increasing trend with increasing velocity. The data indicates that the improvement is related to interphase mass transfer as well as gas phase axial dispersion differences. The interphase mass transfer improvements are evident in the lower velocity range where the conversion efficiency is lower than that of the ideal mixing (CSTR) scenario. The axial dispersion differences are evident above a velocity of $0.3 \mathrm{~m} / \mathrm{s}$ where the MV distributor approaches plug flow performance, while the performance of the baseline distributor remains between the mixing and plug flow extremes (interphase mass transfer is unlikely to play a role at these high velocities since the bed is operating in the turbulent regime).

To determine the transition boundary between the bubbling and turbulent regime two methods were employed namely the maximum standard deviation of the absolute pressure fluctuations in the FBR and visual observation of the bed behaviour [41]. In Figure 8 it can be seen that the maximum of the pressure fluctuations for the baseline case appears to be approximately $0.30 \mathrm{~m} / \mathrm{s}\left(U_{c}\right)$. The MV distributor does not exhibit a maximum within the experimental velocity range, possibly due to signal noise; however from visual observations, using the criteria described by $\mathrm{Bi}$ et al. [44], it was estimated that the turbulent transition takes place between $0.20 \mathrm{~m} / \mathrm{s}$ and 0.30 $\mathrm{m} / \mathrm{s}$. A value of $0.25 \mathrm{~m} / \mathrm{s}$ was subsequently chosen as $U_{c}$ for the MV distributor. The 
value of the transition velocity is also supported by the reaction data where a distinct increase in conversion efficiency is observed at gas superficial velocities between $0.2 \mathrm{~m} / \mathrm{s}$ and $0.3 \mathrm{~m} / \mathrm{s}$. Here the two-phase bubbling bed with interphase mass transfer restrictions transitions into a single phase turbulent bed with axially dispersed plug flow behaviour [32,33]. The $U_{c}$ value for the baseline distributor determined with the pressure fluctuation method was in agreement with the value obtained from the visual observation. A comparative video clip of the distributors at increasing superficial velocities is available to be viewed on YouTube [45] as an upload from the author.

Given the clear differences in interphase mass transfer characteristics, bubble size quantification can assist in understanding these differences. Two separate measurement techniques were employed as discussed in section 2.3 and the respective results are given in Figure 9 and Figure 10. Both sets of results indicate an increase in bubble size as the velocity increases. More interestingly, both sets indicate larger bubbles for the MV distributor which is an unexpected result given the conversion efficiency measurements. Since the standard deviation of the incoherent pressure fluctuations $\sigma_{x y}$, ineed to be calibrated with measured bubbles sizes [34] the bubble growth slope with respect to gas velocity was used to compare the separate techniques. The MV distributor slope was found to be 1.4 times that of the baseline distributor case for the visual measurements while a factor of 1.5 was calculated from the pressure fluctuation measurements. It is therefore evident that the interphase mass transfer difference cannot be attributed to bubble size differences, but rather to the mass transfer mechanism or total bubble fraction in the bed. Total bed height measurements at the same superficial velocity indicated no significant difference between the two distributors and accordingly the total bubble fraction argument is unlikely to explain the observed difference in conversion. From mass transfer correlations, it is know that the mass transfer mechanism consists of a convectional and a diffusional component [46]. One might speculate that the convectional component of the exchange is more severe for the MV bubbles, but no quantification exists to substantiate this argument.

To extend the analysis, the combined bubbling turbulent model of Thompson et al. $[32,33]$ was applied to the system. Table 2 shows the comparative variables for the different studies, with the fitted parameters shown in bold. The column on the far right (light grey) show values fitted by Thomson et al. [32] for experimental results determined by Sun [43] in a 3-D FBR. The measured values for bubble size and transition velocity $\left(U_{c}\right)$ were directly used in the model. The only fitted parameters used are the correction factors for the interphase mass transfer coefficient $\left(f_{k q}\right)$ and axial dispersion $\left(f_{P e}\right)$. With freedom on the value of these two parameters, an error minimisation technique in which the absolute average relative error percentage (AARE\%) were minimised resulted in an adequate representation of the experimental data as shown in Figure 11. For both distributors the error between modelled and actual values was within $10 \%$. The results of the minimisation exercise are shown in Figure 12. The fitted values for $f_{k q}$ show that an increase in mass transfer is apparent; the $f_{k q}$ for the MV distributor is approximately $50 \%$ greater than for the baseline distributor. Given the larger bubbles of the MV distributor this correction is required and can be attributed to the proposed improvement in the mass transfer mechanism. Simulations were run to test the sensitivity of the model to 
changes in $\mathrm{k}_{r}$ "'. The maximum and minimum values of the $\mathrm{kr}^{\prime \prime}$ ' plateau were used and the results are shown in

Figure 13. The band formed gives a maximum error of less than $5 \%$, thus indicating that the major difference between the MV and baseline distributor is not linked to variations in catalyst activity.

The value of $f_{k q}$ for the 3D bed in the Thompson [32,33] study is significantly higher than that of the corresponding 2D scenarios. From geometric considerations (2D vs. 3D) it can be deduced that the factor should be 1.4 times smaller for the 2D scenario, but this is not sufficient to explain the total difference. Another interesting observation is the difference in the axial dispersion correction factor $\left(f_{P e}\right)$. Thompson uses the correlation of $\mathrm{Bi}$ et al. [44] for the prediction of axial dispersion in the turbulent regime. For the two dimensional bed in this study a vessels Peclet number of approximately 4 is predicted by this correlation, suggesting that the behaviour of the turbulent bed will be somewhere between ideal mixing and plug flow. The correction factor for the baseline 2D distributor in this study as well as the one in the Thompson study [32] indicates that the fitted dispersion is roughly in agreement with the correlation, while this is not nearly the case for the MV distributor where near plug flow behaviour $(P e \simeq 6)$ is observed at a velocity of $0.35 \mathrm{~m} / \mathrm{s}$. Similar to the interphase mass transfer difference the reason for the major improvement in vessel Peclet number can only be speculated on.

Lastly one should quantify the interplay between interphase mass transfer and axial dispersion. This was done by varying the two fitted parameters while monitoring the error. From Figure 12 it is evident that variation in $f_{P e}$ as well as $f_{k q}$ is limited to a range of values. The narrow band of the $f_{k q}$ parameter is not linked to the value of the $f_{P e}$ parameter, indicating the independence between mass transfer and axial dispersion where $f_{k q}$ affects the bubbling regime while $f_{P e}$ only affects the tail end of the prediction (turbulent regime) where the model transitions from the two phase model to the axially dispersed plug flow model and the designated asymptote between the CSTR and PFR extremes is set by the value of $f_{P e}$.

\section{Conclusions}

The pseudo-2D FBR performance using a multi-vortex (MV) distributor was compared to a perforated plate (baseline) distributor with comparable open area and superficial velocity. In this study the ozone decomposition reaction in the bed was used to determine the overall performance of the distributors and it was found that the MV distributor causes a significant improvement in the conversion efficiencies at all velocities tested. The improvement varied between $0 \%$ and $20 \%$, with a mean improvement of $14.7 \%$ and an increasing trend with increasing velocity.

Additionally the bubble sizes were measured using a visual measurement technique as well as a pressure fluctuation analysis technique. The bubbles were found to grow with superficial velocity at a rate approximately 1.4 times greater for the MV distributor than the baseline case. The bubbling to turbulent transition boundary $\left(U_{c}\right)$ was determined visually and by measuring the standard deviation of the pressure fluctuations in the bed, the MV distributor did not exhibit a maximum standard deviation, but from visual observations the $U_{c}$ was determined to be at $0.25 \mathrm{~m} / \mathrm{s}$ and 
was supported by reaction data. The baseline distributor exhibited a maximum standard deviation at $0.3 \mathrm{~m} / \mathrm{s}$, which was supported by visual observation.

The improved overall performance for the MV distributor as compared to the baseline, despite the increase in bubble sizes, could indicate that the mass transfer mechanism as opposed to the actual bubble sizes influence the performance, i.e. a possible convective component to the mass transfer for the MV bed, which exceeds that of the baseline could be present, but no quantification is available to support the validity of this argument. The conversion for the MV distributor increases to near plug flow at velocities exceeding $U_{c}$, which indicates a significant decrease in the axial dispersion in the bed; the predicted $P e \simeq 6$ for the MV distributor at these velocities as compared to the baseline $P e \simeq 3$.

\section{Nomenclature}

\begin{tabular}{|c|c|c|}
\hline$A_{\text {open }}$ & Total open area on distributor & $\mathrm{mm}^{2}$ \\
\hline$A_{o r}$ & Injection orifice cross-sectional area & $\mathrm{mm}^{2}$ \\
\hline$C_{\text {ozone, IN }}$ & Ozone inlet concentration & $\mathrm{mg} / \mathrm{L}$ \\
\hline$C_{\text {ozone, OUT }}$ & Ozone outlet concentration & $\mathrm{mg} / \mathrm{L}$ \\
\hline$d_{p}$ & Average particle size & $\mathrm{m}$ \\
\hline$f_{c}$ & Fraction of total solids in cyclones and dipleg during normal operation & - \\
\hline$f_{k q}$ & Interphase mass transfer fitting parameter & - \\
\hline$f_{P e}$ & Peclet number fitting parameter & - \\
\hline$I D$ & Inner diameter of injection orifice & $\mathrm{mm}$ \\
\hline$k_{r}^{\prime \prime}$ & Reaction rate constant & $s^{-1}$ \\
\hline Mso & Total solids inventory in bed & $\mathrm{kg}$ \\
\hline$N_{o r}$ & Number of injection orifices & - \\
\hline$P e$ & Vessels Peclet number & - \\
\hline Q & Volumetric flowrate into the reactor & $\mathrm{m}^{3} / \mathrm{s}$ \\
\hline$U_{0}$ & Linear superficial inlet velocity & $\mathrm{m} / \mathrm{s}$ \\
\hline$U_{c}$ & Bubbling to turbulent regime transition velocity & $\mathrm{m} / \mathrm{s}$ \\
\hline$U_{o r}$ & Orifice superficial velocity & $\mathrm{m} / \mathrm{s}$ \\
\hline$V$ & Volume of the reactor & $\mathrm{m}^{3}$ \\
\hline$x$ & Conversion of ozone to oxygen & - \\
\hline$\mu_{g}$ & Gas viscosity & a.s \\
\hline
\end{tabular}




\begin{tabular}{|c|c|c|}
\hline$\rho_{g}$ & Gas density & $\mathrm{kg} / \mathrm{m}^{3}$ \\
\hline$\rho_{s}$ & Solid particle density & $\mathrm{kg} / \mathrm{m}^{3}$ \\
\hline$\sigma_{\text {ozone, IN }}$ & Standard deviationin $C_{\text {ozone, IN }}$ & $\mathrm{mg} / \mathrm{L}$ \\
\hline$\sigma_{\text {ozone,OUT }}$ & Standard deviation in $C_{\text {ozone,OUT }}$ & $\mathrm{mg} / \mathrm{L}$ \\
\hline$\sigma_{x}^{2}$ & Variance in the conversion of ozone to oxygen & - \\
\hline$\sigma_{x y, i}$ & The incoherent standard deviation of pressure fluctuations [33] & $\mathrm{kPa}$ \\
\hline
\end{tabular}

\section{Acknowledgement}

The authors gratefully acknowledge THRIP for the financial support for this work.

\section{References}

[1] J.R. Van Ommen, J. Nijenhuis, M.O. Coppens, Reshaping the Structure of Fluidized Beds, CEP. (2009) 49-57.

[2] L. Shen, F. Johnsson, B. Leckner, Digital image analysis of hydrodynamics two-dimensional bubbling fluidized beds, Chemical Engineering Science. 59 (2004) 2607-2617.

[3] M. Köksal, H. Vural, Bubble size control in a two-dimensional fluidized bed using a moving double plate distributor, Powder Technology. 95 (1998) 205213.

[4] P.N. Rowe, L. Santoro, J.G. Yates, The division of gas between bubble and interstitial phases in fluidised beds of fine powders, Chem. Eng. Sci. 33 (1978) 133.

[5] D. Geldart, R.R. Cranfield, The gas fluidisation of large particles, Chemical Engineering Journal. 3 (1972) 211.

[6] C. Sobrino, A. Acosta-lborra, D. Santana, M. De Vega, Bubble characteristics in a bubbling fluidized bed with a rotating distributor, Int. J. Multiphase Flow. 35 (2009) 970-976.

[7] C.S. Chyang, Y.C. Lin, A study in the swirling fluidizing pattern, J. Chem. Eng. Jpn. 35 (2002) 503-512.

[8] B. Sreenivasan, V.R. Raghavan, Hydrodynamics of a swirling fluidised bed, Chemical Engineering and Processing. 41 (2002) 99-106.

[9] C.S. Chyang, K. Lieu, S.S. Hong, The effect of distributor design on gas dispersion in a bubbling fluidized bed, Journal of the Chinese Institute of Chemical Engineers. 39 (2008) 685-692.

[10] M. Wormsbecker, T.S. Pugsley, H. Tanfara, The Influence of Distributor Design on Fluidized Bed Dryer Hydrodynamics, in: The 12th International Conference on Fluidization-New Horizons in Fluidization Engineering, 2007: p. 100.

[11] F. Kleijn van Willigen, D. Christensen, J.R. Van Ommen, M.O. Coppens, Imposing dynamic structures on fluidised beds, Catalysis Today. 105 (2005) 560-568. 
[12] H.W. Wong, M.H.I. Baird, Fluidisation in a pulsed gas flow, The Chemical Engineering Journal. 2 (1971) 104-113.

[13] D.V. Pence, D.E. Beasley, Chaos suppression in gas-solid fluidization, Chaos. 8 (1998) 514-519.

[14] F. Kleijn van Willigen, J.R. Van Ommen, J. Van Turnhout, C.M. Van Den Bleek, Bubble size reduction in a fluidized bed by electric fields, International Journal of Chemical Reaction Engineering. 1 (2003) A21.

[15] G. Sun, J. Grace, The effect of particle size distribution on the performance of a catalytic fluidized bed reactor, Chemical Engineering Science. 45 (1990) 21872194.

[16] R. Beetstra, J. Nijenhuis, N. Ellis, J.R. van Ommen, The influence of the particle size distribution on fluidized bed hydrodynamics using high-throughput experimentation, AIChE J. 55 (2009) 2013-2023.

[17] R. Samson, C.E. Brakel, A.M. Scott, K. Chandrasekharan, P. Veenstra, A bubble model describing the influence of internals on gas fluidization, Chemical Engineering Science. 43 (1988) 2215-2220.

[18] I. Miracca, G. Capone, The staging in fluidised bed reactors: from CSTR to plug-flow, Chemical Engineering Journal. 82 (2001) 259-266.

[19] J.J. Van Dijk, A.C. Hoffmann, D. Cheesman, J.G. Yates, The influence of horizontal internal baffles on the flow pattern in dense fluidized beds by X-ray investigation, Powder Technology. 98 (1998) 273-278.

[20] D.S. Sathiyamoorthy, C.S. Rao, Gas distributor in fluidized beds, Powder Technol. 20 (1978) 47-52.

[21] J.G. Yates, P.N. Rowe, D.J. Cheesman, Gas entry effects in fluidized bed reactors, AIChE Journal. 30 (1984) 890-893.

[22] C. Sobrino, N. Ellis, M. De Vega, Distributor effects near the bottom region of turbulent fluidized beds, Powder Technol. 189 (2009) 25-33.

[23] D. Carmello, D.B. Garrod, M. Pardocchi, M. Ioppi, P.U. Foscolo, Horizontal gas mixing in the distributor region of a fluidised bed reactor, Chemical Engineering Journal. 76 (2000) 153-158.

[24] J.F. Davidson, R.B. Thorpe, M. Pollitt, J. Smith, Maldistribution in fluidized beds, Ind. Eng. Chem. Res. 41 (2002) 5878-5889.

[25] N. Ellis, H.T. Bi, C.J. Lim, J.R. Grace, Hydrodynamics of turbulent fluidized beds of different diameters, Powder Technology. 141 (2004) 124-136.

[26] A. Yan, W. Huang, J. Zhu, The influence of distributor structure on the solid distribution and flow development in circulating fluidized beds, Can. J, Chem. Eng. 86 (2008) 1023-1031.

[27] F. Ouyang, O. Levenspiel, Spiral distributor for fluidized beds, Industrial \& Engineering Chemistry Process Design and Development. 25 (1986) 504-507.

[28] C.S. Chyang, F.P. Qian, H.Y. Chiou, Radial Gas Mixing in a Fluidized Bed with a Multi-Horizontal Nozzle Distributor using Response Surface Methodology, Chem. Eng. Technol. 30 (2007) 1700-1707.

[29] J. De Wilde, A. de Broqueville, Rotating fluidized beds in a static geometry: Experimental proof of concept, AIChE J. 53 (2007) 793-810.

[30] C.E. Dodson, V.I. Lakshmanan, An innovative gas-solid torbed reactor for the recycling industries, JOM Journal of the Minerals, Metals and Materials Society. 50 (1998) 29-31.

[31] O. Bolland, R. Nicolai, Describing mass transfer in circulating fluidized beds by ozone decomposition, Chemical Engineering Communications. 187 (2001) 121. 
[32] M.L. Thompson, H.T. Bi, J.R. Grace, A generalized bubbling/turbulent fluidized-bed reactor model, Chem. Eng. Sci. 54 (1999) 2175-2185.

[33] I.A. Abba, J.R. Grace, H.T. Bi, M.L. Thompson, Spanning the flow regimes: generic fluiidized-bed reactor model, AIChE Journal. 49 (2003) 1838-1848.

[34] J. Van der Schaaf, J.C. Schouten, F. Johnsson, C.M. Van den Bleek, Nonintrusive determination of bubble and slug length scales in fluidized beds by decomposition of the power spectral density of pressure time series, International Journal of Multiphase Flow. 28 (2002) 865-880.

[35] J. Saayman, Bubbling to Turbulent Regime Transition in a 2D Catalytic Fluidized Bed Reactor, Masters Thesis, University of Pretoria, 2009.

[36] S.B. Reddy Karri, J. Werther, Gas Distributor and Plenum Design in Fluidized Beds, in: W.C. Yang (Ed.), Handbook of Fluidization and Fluid-Particle Systems, Marcel Dekker, Inc., New York, 2003: pp. 155-170.

[37] H.S. Fogler, Elements of Chemical Reaction Engineering, Fourth Edition, Pearson Education Inc., USA, 2006.

[38] S. Ouyang, J. Lin, O.E. Potter, Ozone decomposition in a 0.254 mdiameter circulating fluidized bed reactor., Powder Technology. 74 (1993) 73.

[39] S. Zimmermann, F. Taghipour, CFD modeling of the hydrodynamics and reaction kinetics of FCC fluidized-bed reactors, Ind. Eng. Chem. Res. 44 (2005) 9818-9827.

[40] C. Fan, Y. Zhang, X. Bi, W. Song, W. Lin, L. Luo, Evaluation of downer reactor performance by catalytic ozone decomposition, Chemical Engineering Journal. 140 (2008) 539-554.

[41] H. Schoenfelder, M. Kruse, J. Werther, Two-dimensional model for circulating fluidized-bed reactors, AIChE Journal. 42 (1996) 1875-1888.

[42] S. Pagliolico, M. Tiprigan, G. Rovero, A. Gianetto, Pseudo-homogeneous approach to CFB reactor design, Chemical Engineering Science. 47 (1992) 2269-2274.

[43] G. Sun, Influence of particle size distribution on the performance of fluidized bed reactors., Ph.D. thesis, University of British Columbia, 1991.

[44] H.T. Bi, N. Ellis, J.R. Grace, A state-of-the-art review of gas-solid turbulent fluidization, Chemical Engineering Science. 55 (2000) 4789-4825.

[45] H.G. Brink, YouTube - Perforated plate and Multi vortex distributor in a 2D fluidized bed reactor compared

(http://www.youtube.com/watch?v=qWH4MHR4IQo) accessed: [2011-05-30 14:14:34], (2011).

[46] S.P. Sit, J.R. Grace, Effect of Bubble Interaction on Interphase Mass Trnasfer in Gas Fluidized Beds, Chem. Eng. Sci. 36 (1981) 327-335.

\section{Captions:}

Table 1: Comparative distributor characteristics for the experimental study

Table 2: The Variables used for the simulation of the Thompson model; the fitted parameters are shown in dark grey

Figure 1: The piping and instrumentation for the experimental setup adapted from Saayman [35]. 
Figure 2: Photograph and front-view representation of the gas flow pattern of the MV distributor during operation

Figure 3: The measured $-\ln (1-x)$ vs. V/Q data points for different experimental runs, measured in the packed bed reactor in parallel to the FBR. The slope of the data is equal to the first order $k_{r}$ "' and can be seen to be limited between $1.2 \mathrm{~s}^{-1}$ and $1.8 \mathrm{~s}^{-1}$.

Figure 4: Typical curve for the deactivation of a single catalyst sample over time.

Figure 5: Typical range of snapshots of the bubble sizes taken $\left(U_{0}=0.2 \mathrm{~m} / \mathrm{s}, \mathrm{MV}\right.$ distributor).

Figure 6: The average conversion efficiency for the measured conversions. Error bars show one standard deviation from the average.

Figure 7: a) The moving average of the measured conversion efficiencies for the MV distributor and the baseline distributors, taken over 5 consecutive data points. b) The percentage improvement from the baseline to MV distributor

Figure 8: The standard deviation of the pressure fluctuations of both the baseline and MV distributor experimental runs.

Figure 9: a) and b) The visually measured bubble diameters with the linear correlation of the bubble diameters for the baseline and MV distributor respectively.

c) The comparison of the best fit linear correlations from a) and b)

Figure 10: The $\sigma_{x y, i}$ of the baseline and the MV distributor used for the analysis of the bubble sizes in the separate experimental studies.

Figure 11: a): The Thompson model predictions for the measured conversion efficiencies for both the baseline and the MV distributor. Also shown is the CSTR model for the respective distributor cases. b) and c): The \% Error plots for the baseline and MV distributor.

Figure 12: Contour plots showing the average absolute relative error percentages (AARE\%) of the Thompson model with respect to the experimental results for different values of the fitting parameters $\mathrm{f}_{\mathrm{kq}}$ andf $\mathrm{Pe}_{\mathrm{Pe}}$. The plot on the left shows the optimal solution for the baseline case and the right shows the solution for the MV case.

Figure 13: Effect of $k_{r}$ "' on conversion efficiency. The maximum error is less than $5 \%$. 
Table 1: Comparative distributor characteristics for the experi

Baseline Distributor MV Distributor

\begin{tabular}{lrr}
\hline$I D(m m)$ & 1.9 & 1.755 \\
$A_{\text {or }}\left(\mathrm{mm}^{2}\right)$ & 2.84 & 2.42 \\
$N_{\text {or }}$ & 35 & 38 \\
$A_{\text {open }}\left(\mathrm{mm}^{2}\right)$ & 99 & 92 \\
\hline$U_{\text {or }} / U_{\circ}$ & 101 & 109 \\
\hline
\end{tabular}




\begin{tabular}{|c|c|c|c|}
\hline Variable & \multicolumn{2}{|c|}{ Current Study } & Thompson et al.[31] \\
\hline Distributor & Perforated Plate & MV Distributor & Perforated Plate \\
\hline Column Type & $2-D$ & $2-D$ & $3-D$ \\
\hline$k_{r}^{\prime \prime \prime}$ & $1.6 \mathrm{~s}^{-1}$ & $1.33 \mathrm{~s}^{-1}$ & $2.41 \mathrm{~s}^{-1}$ \\
\hline$M_{\text {so }}$ & 3.6 & 3.6 & 5 \\
\hline Catalyst & FCC & FCC & FCC \\
\hline$d_{p}$ & $87 \mu \mathrm{m}$ & $84 \mu \mathrm{m}$ & $60 \mu \mathrm{m}$ \\
\hline Bubble size & $2.3 \mathrm{~cm}-5.1 \mathrm{~cm}$ & $2.2 \mathrm{~cm}-7.7 \mathrm{~cm}$ & $8 \mathrm{~cm}$ \\
\hline $\boldsymbol{f}_{c}$ & 0.2 & 0.2 & 0.2 \\
\hline$f_{k q}$ & 0.48 & 0.73 & 2.023 \\
\hline$f_{P e}$ & 0.66 & 1.47 & 0.247 \\
\hline$\Phi_{L O}$ & $3.55 \%$ & $3.55 \%$ & $3.55 \%$ \\
\hline$\mu_{g}$ & $2.0 \times 10^{-5} \mathrm{~Pa} . \mathrm{s}$ & $2.0 \times 10^{-5} \mathrm{~Pa} . \mathrm{s}$ & $2.0 \times 10^{-5} \mathrm{~Pa} . \mathrm{s}$ \\
\hline$\rho_{s}$ & $1580 \mathrm{~kg} / \mathrm{m}^{3}$ & $1580 \mathrm{~kg} / \mathrm{m}^{3}$ & $1580 \mathrm{~kg} / \mathrm{m}^{3}$ \\
\hline$\rho_{g}$ & $1.20 \mathrm{~kg} / \mathrm{m}^{3}$ & $1.20 \mathrm{~kg} / \mathrm{m}^{3}$ & $1.20 \mathrm{~kg} / \mathrm{m}^{3}$ \\
\hline
\end{tabular}




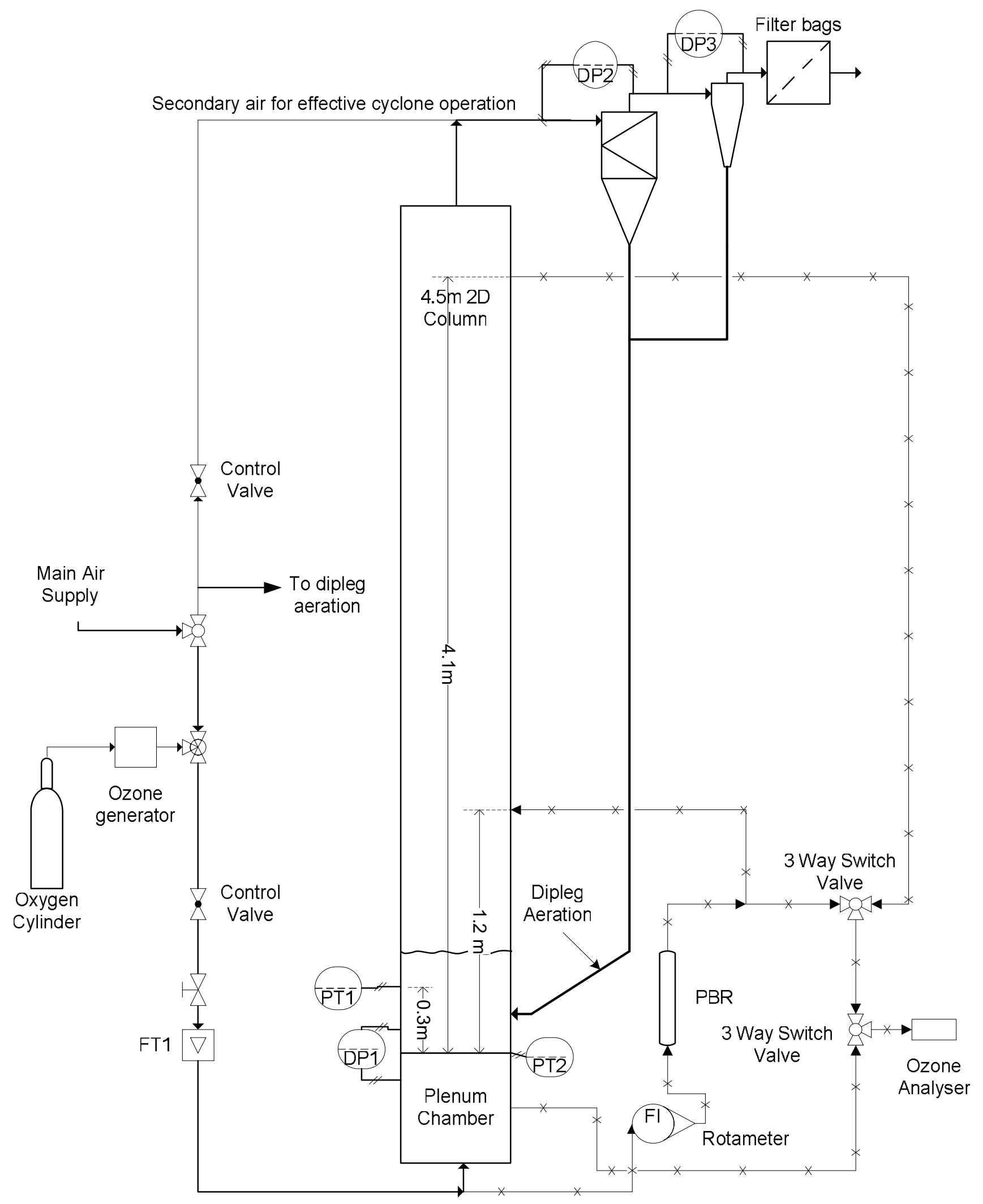




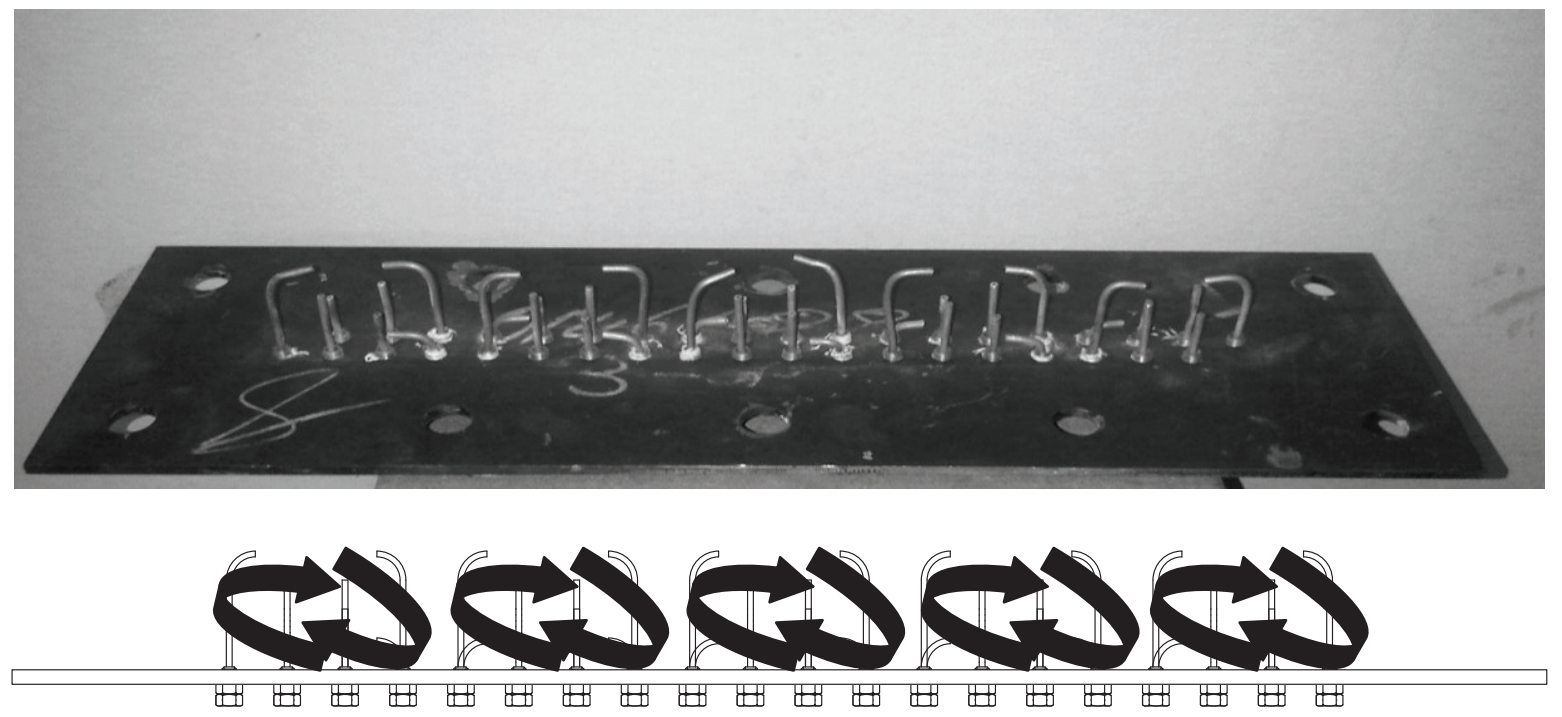

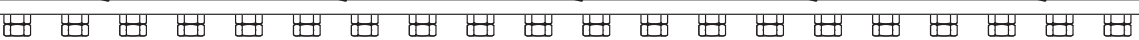


Figure 3: The measured $-\ln (1-x)$ vs. V/Q data points for differen

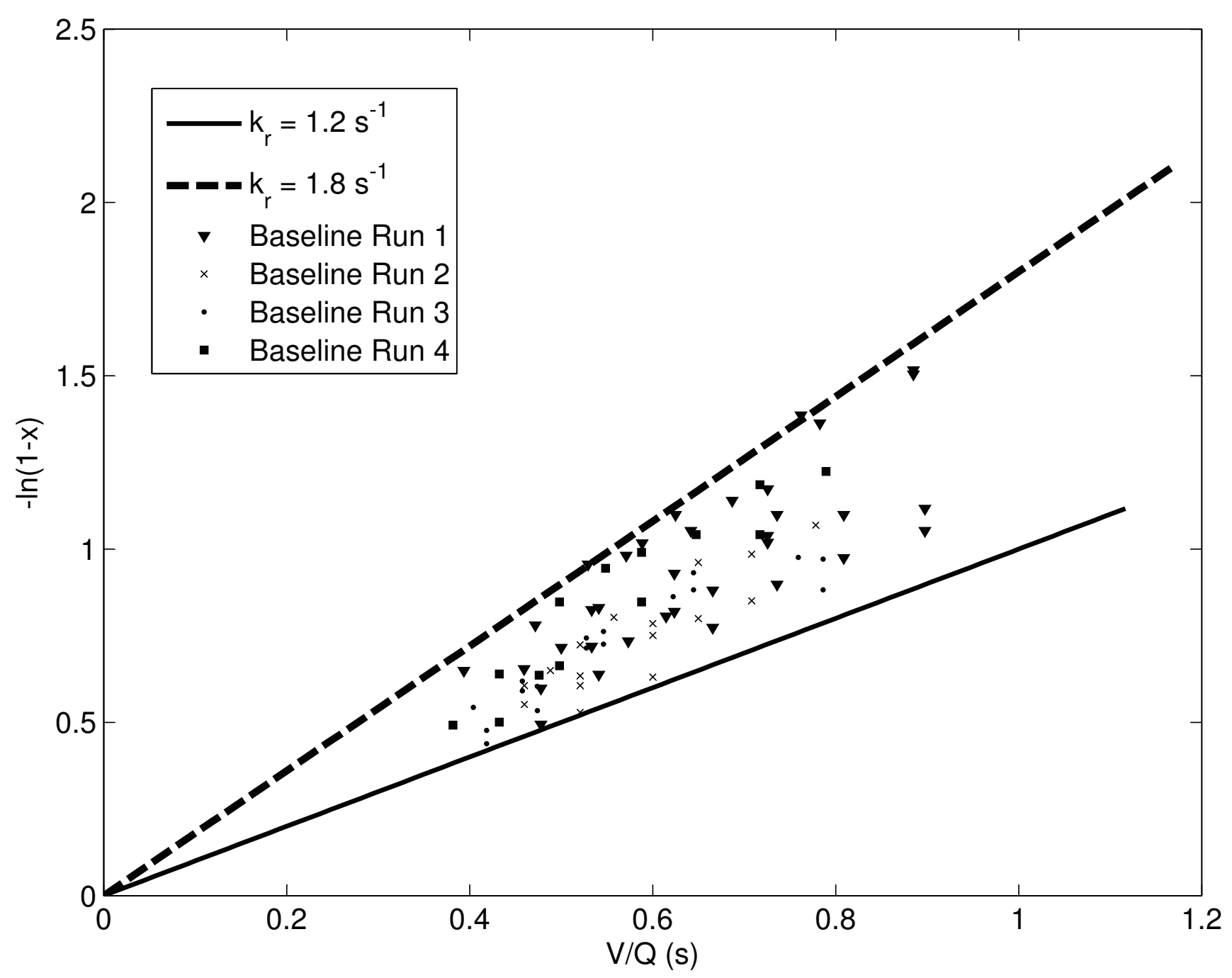


Figure 4: Typical curve for the deactivation of the catalyst

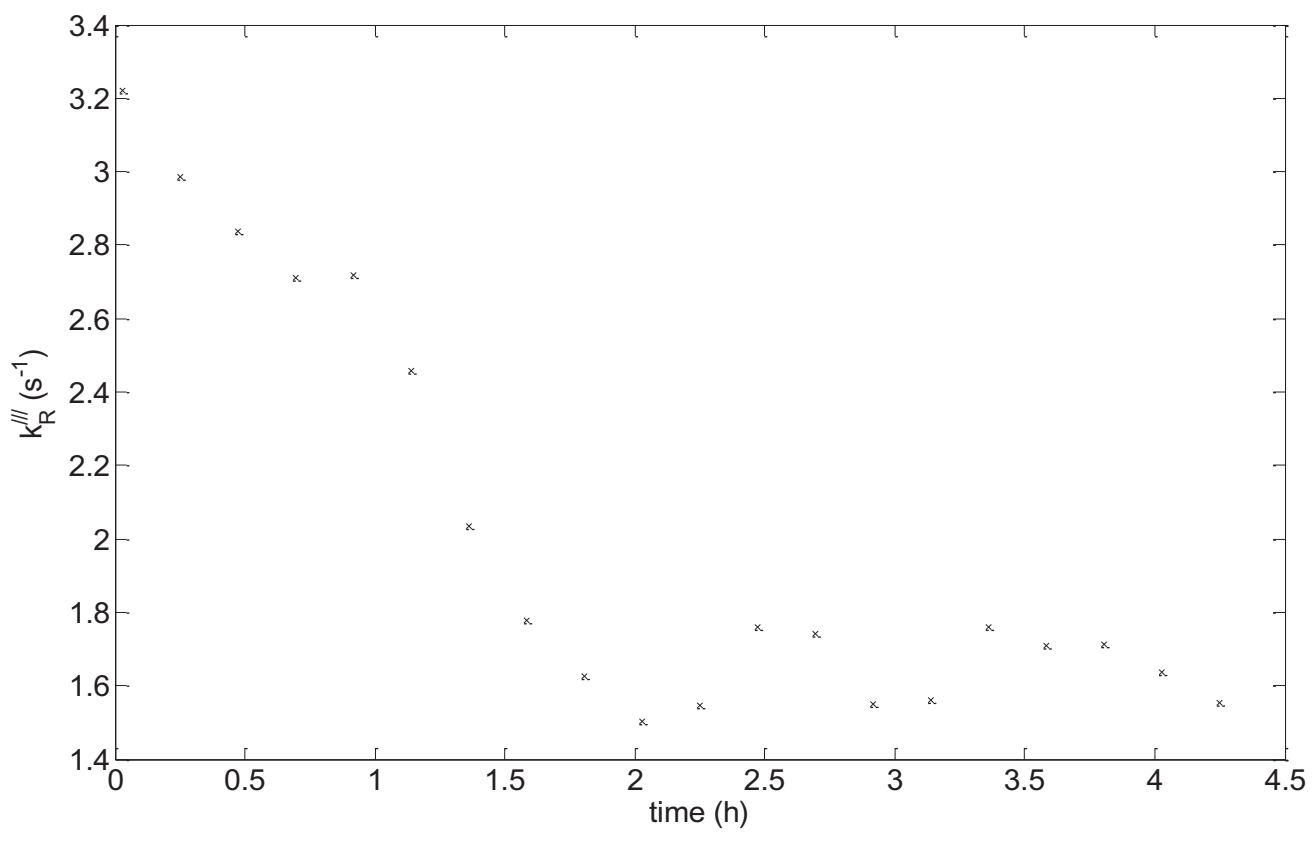




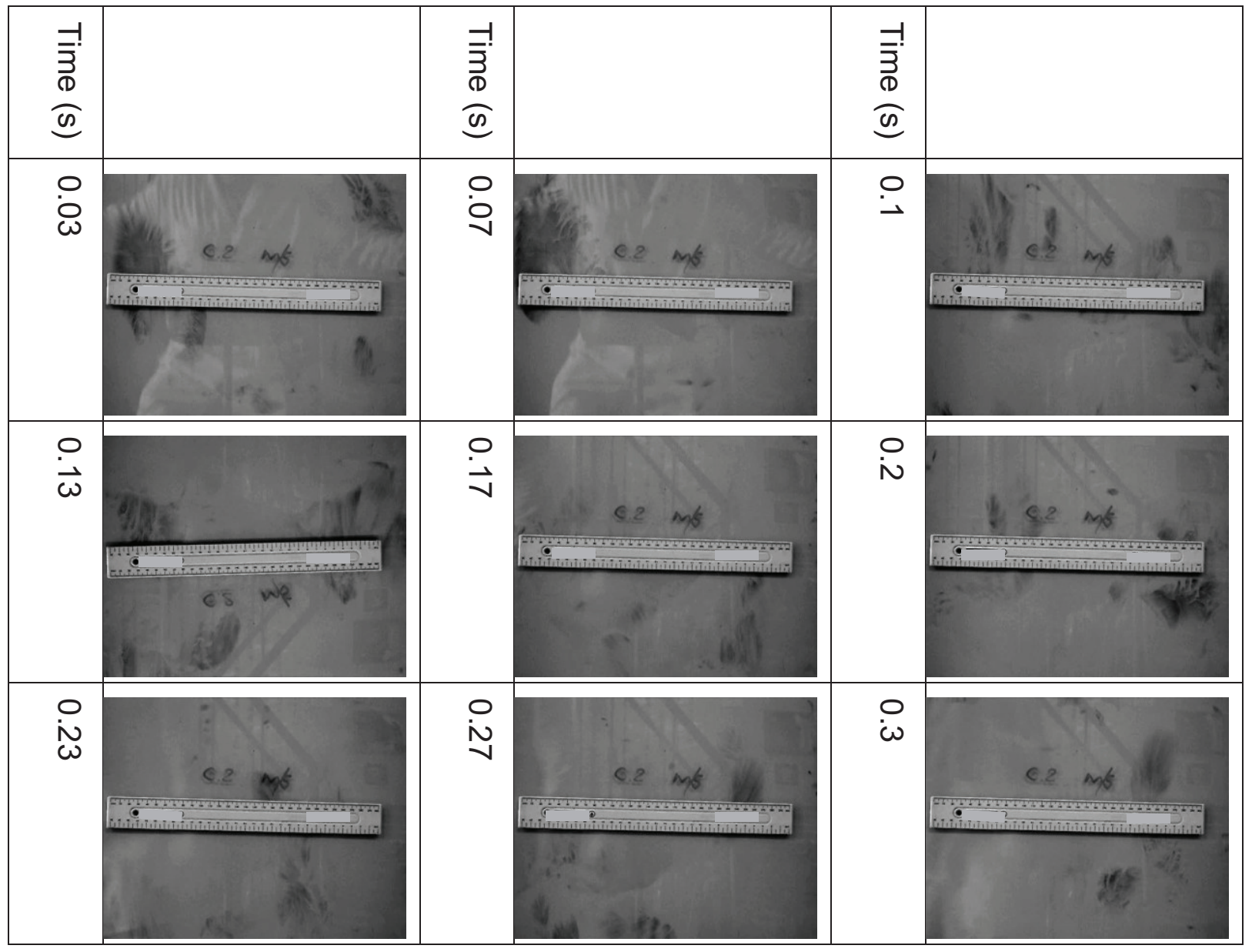



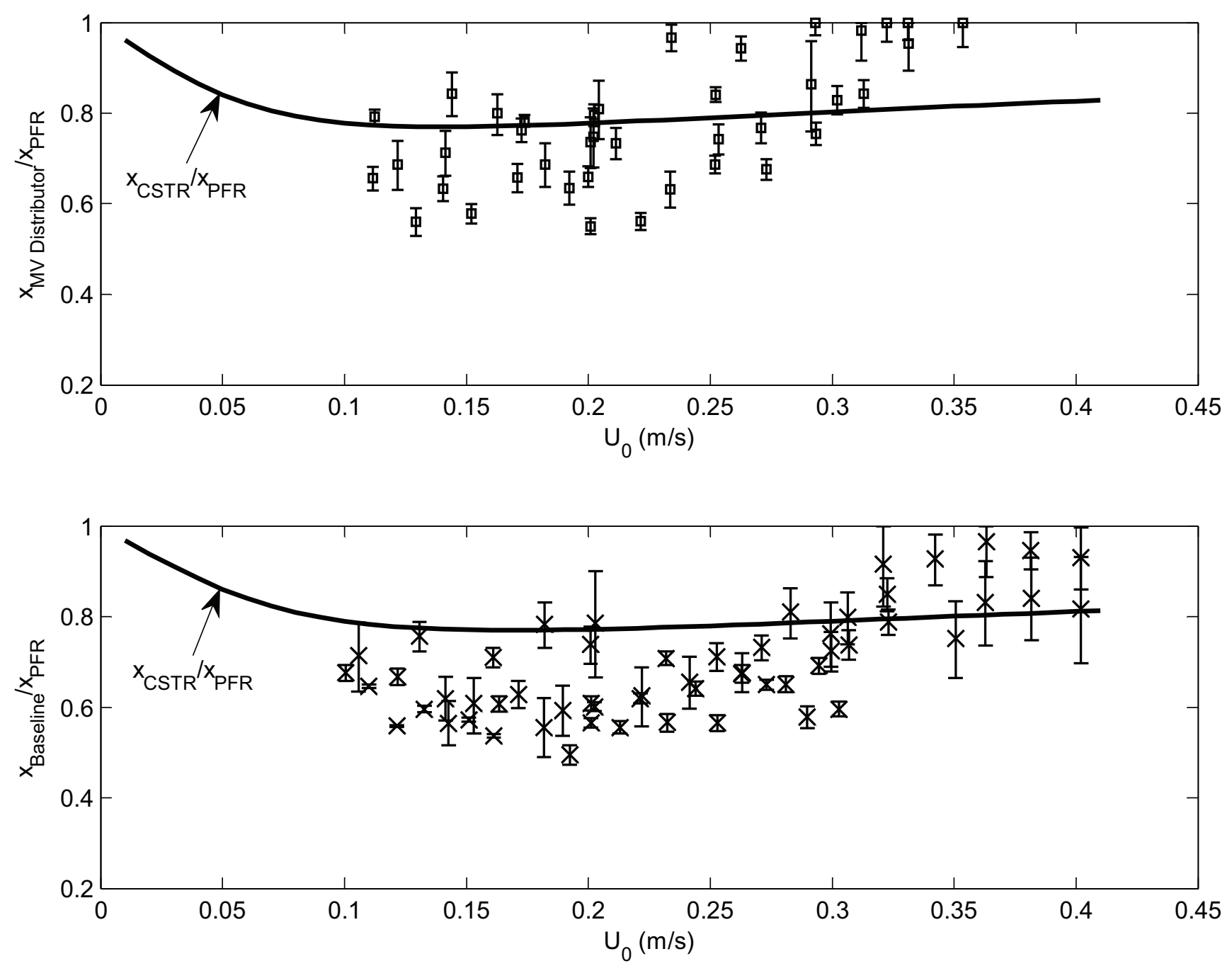
Figure 7: a) The moving average of the measured conversion
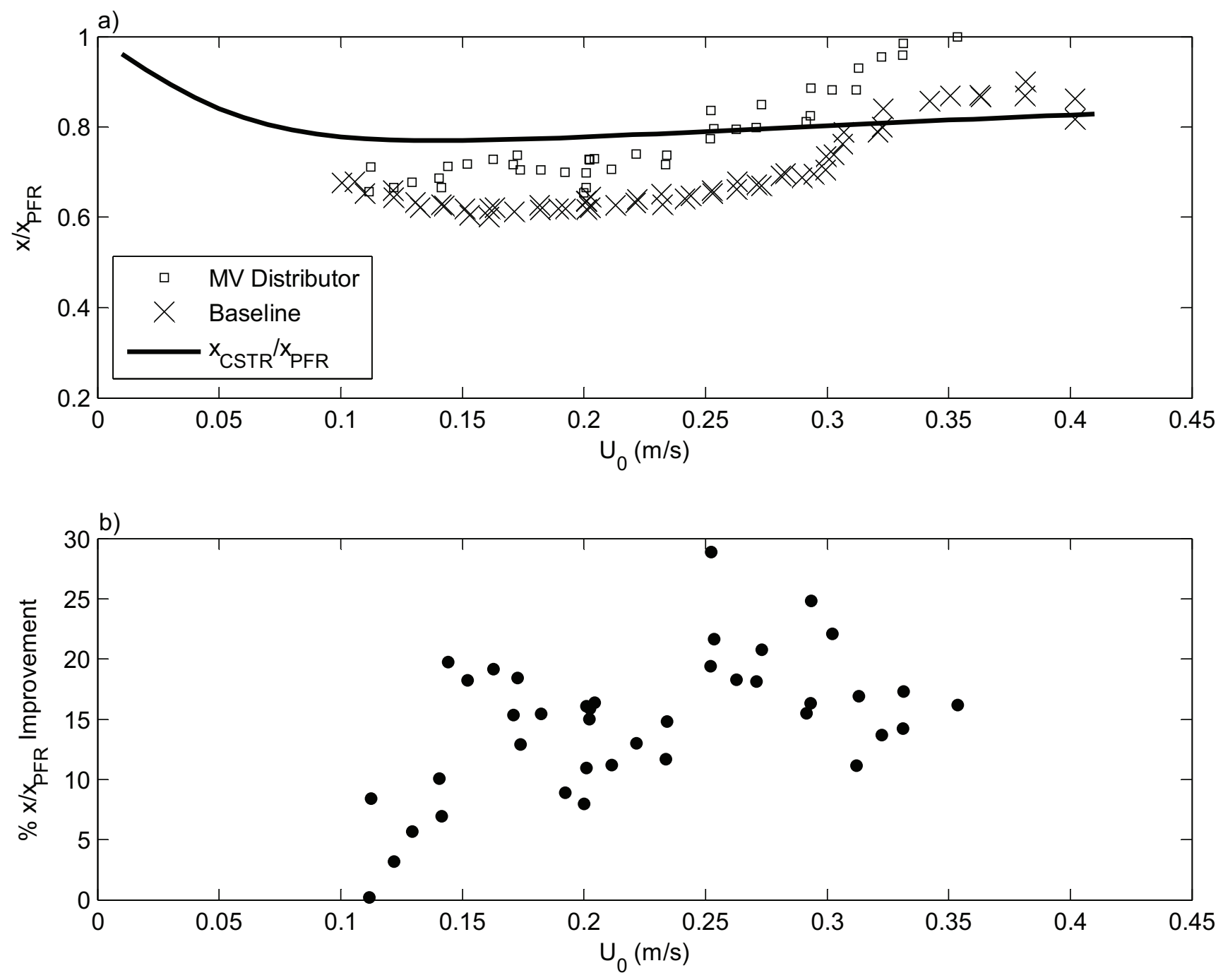
Figure 8: The standard deviation of the pressure fluctuations

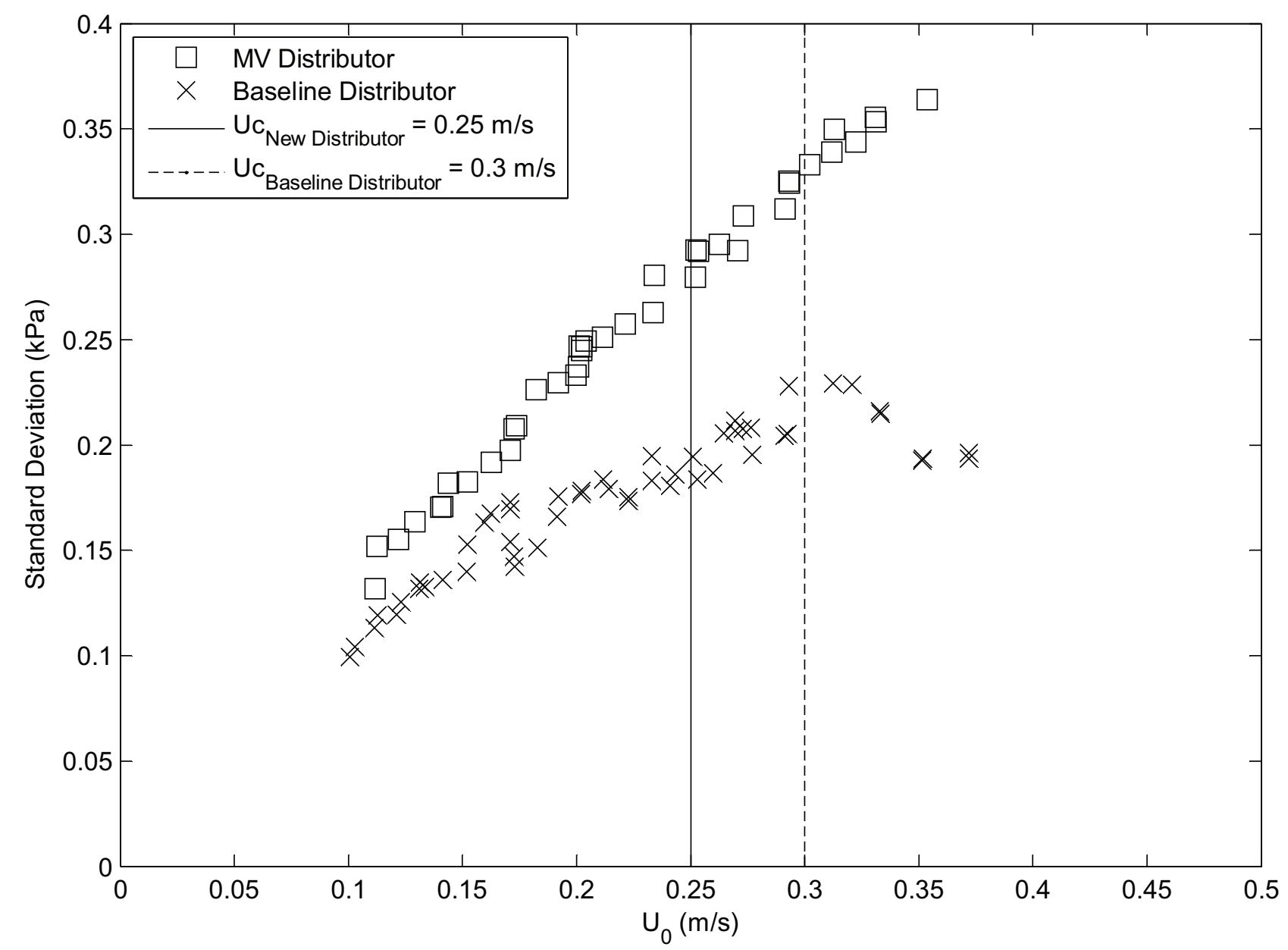



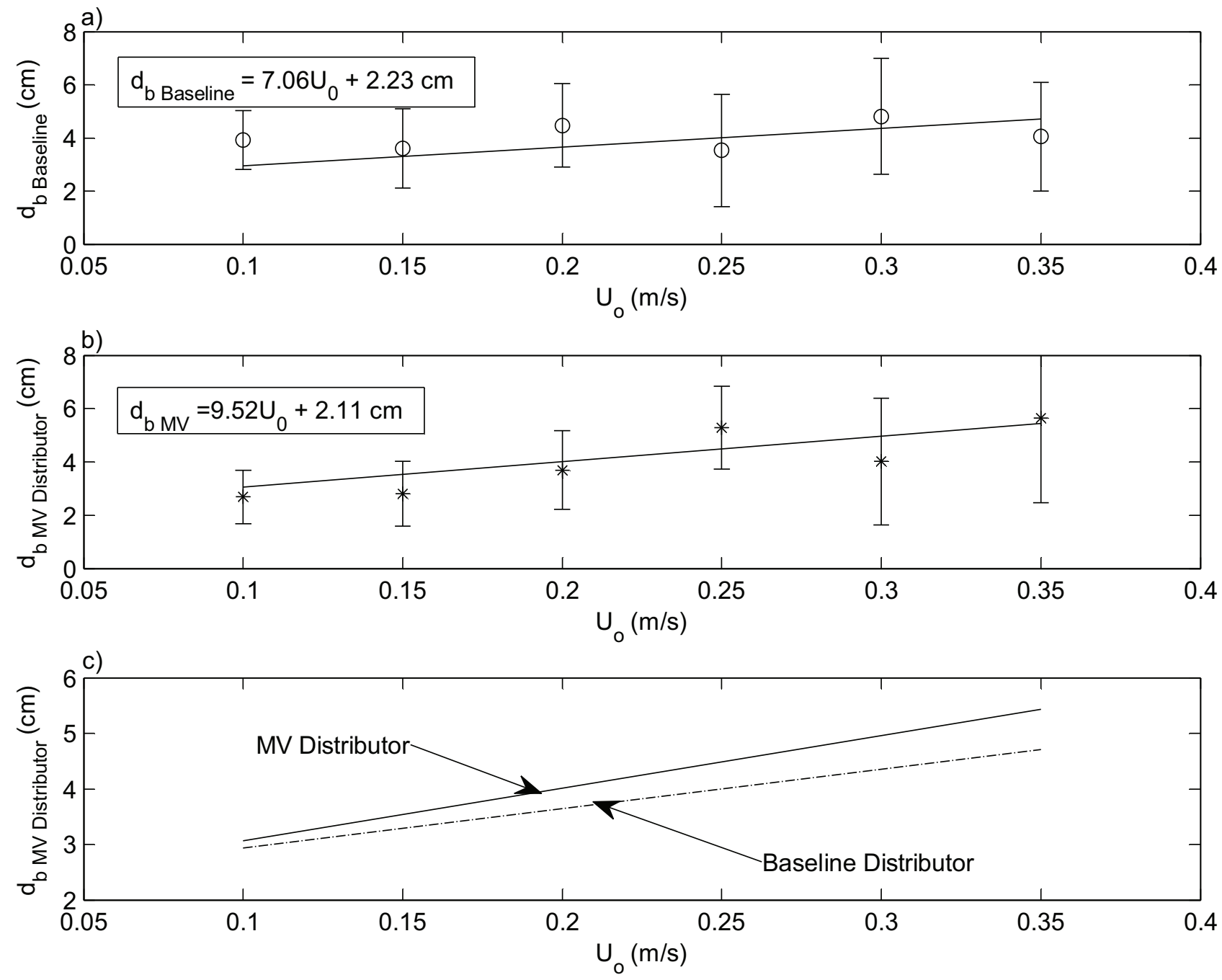


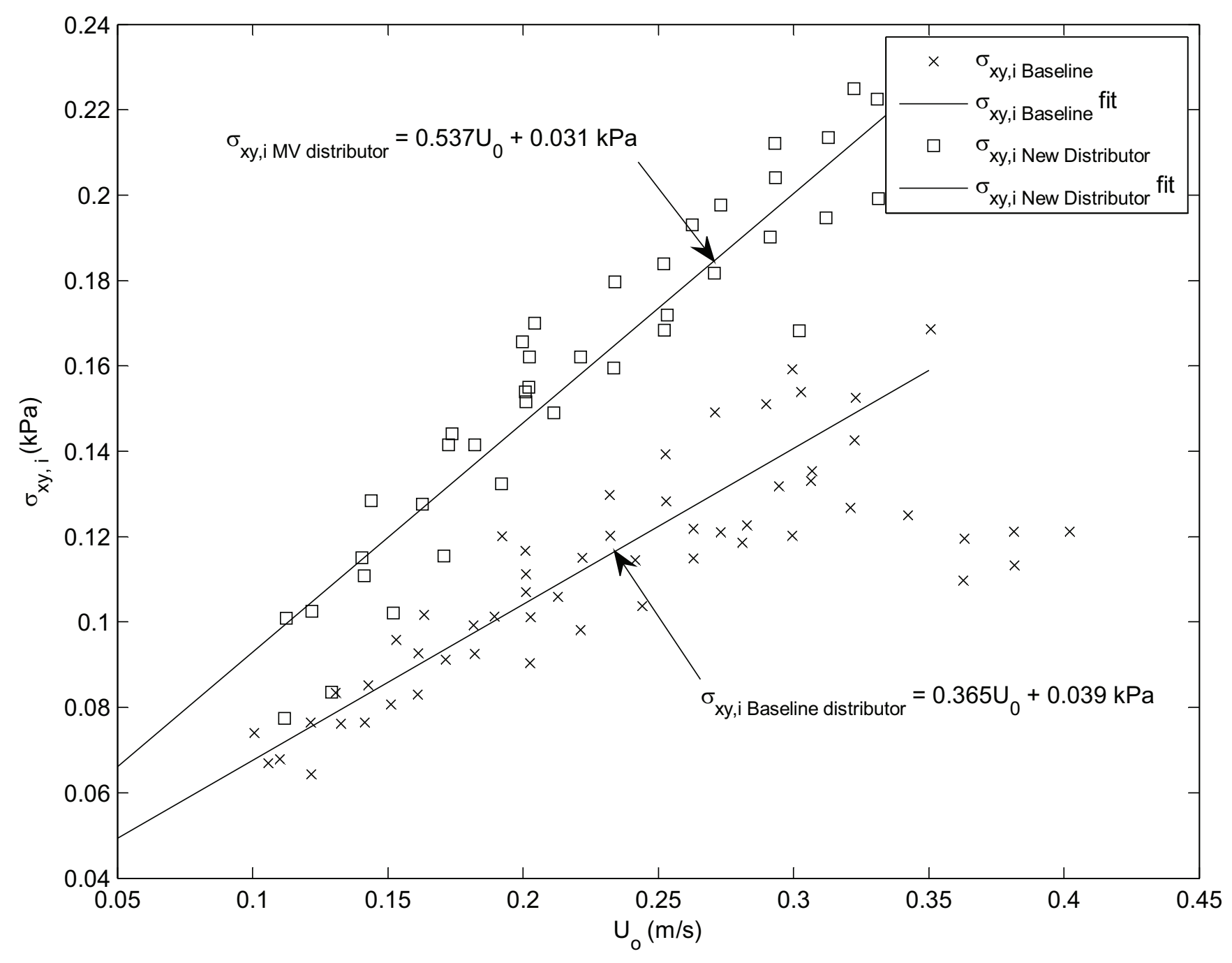



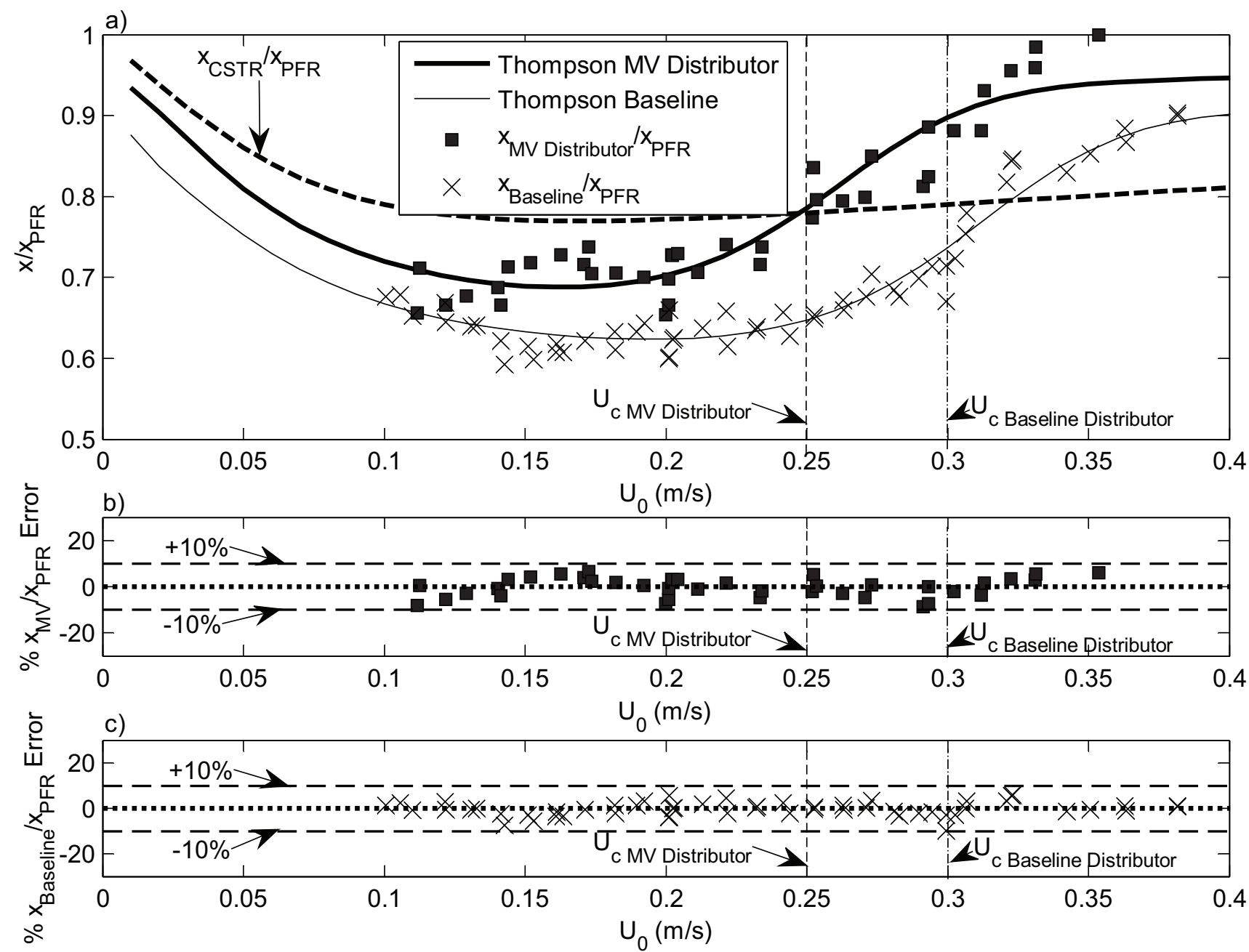

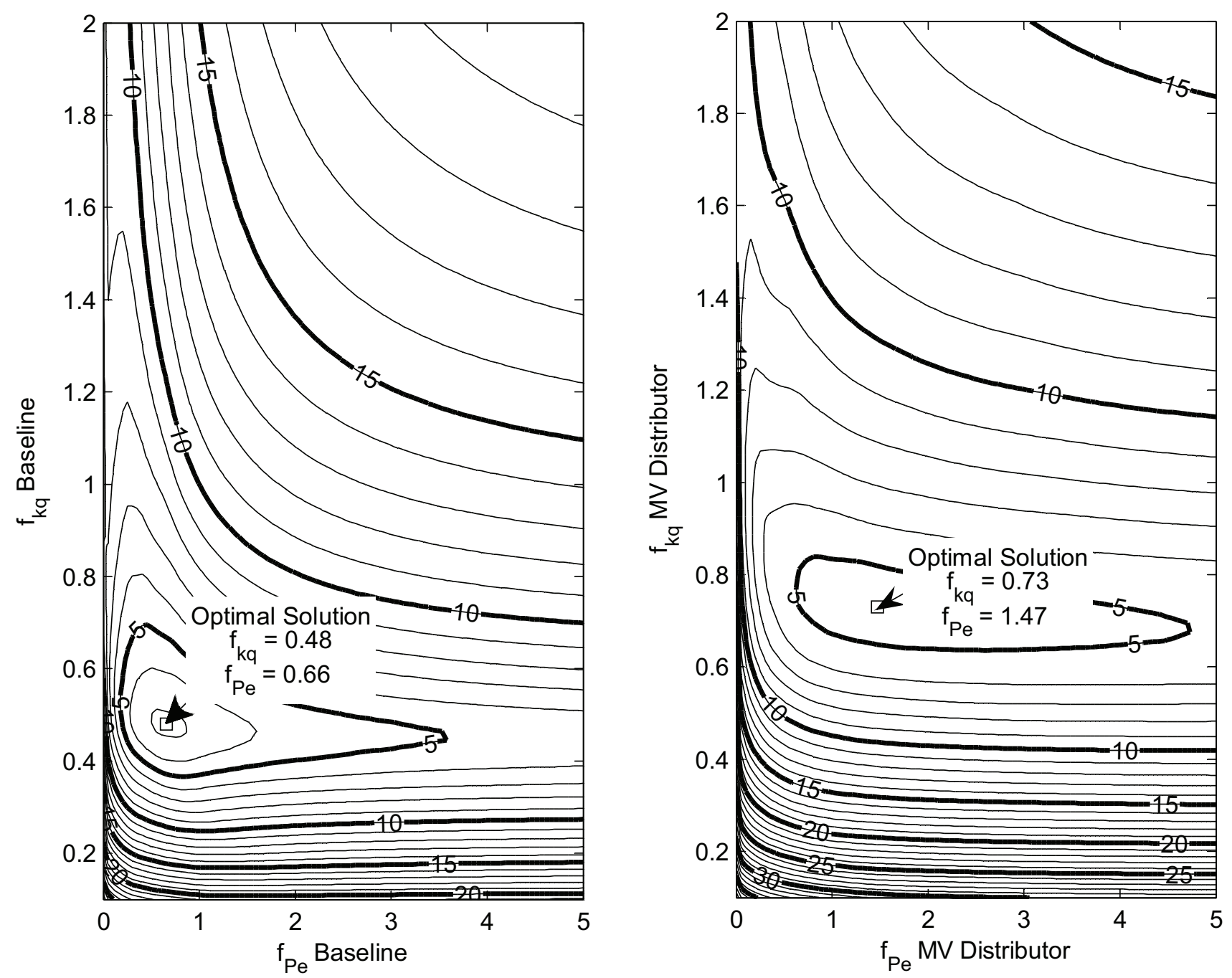
Figure 13: Effect of kr"' on conversion efficiency. The maximum

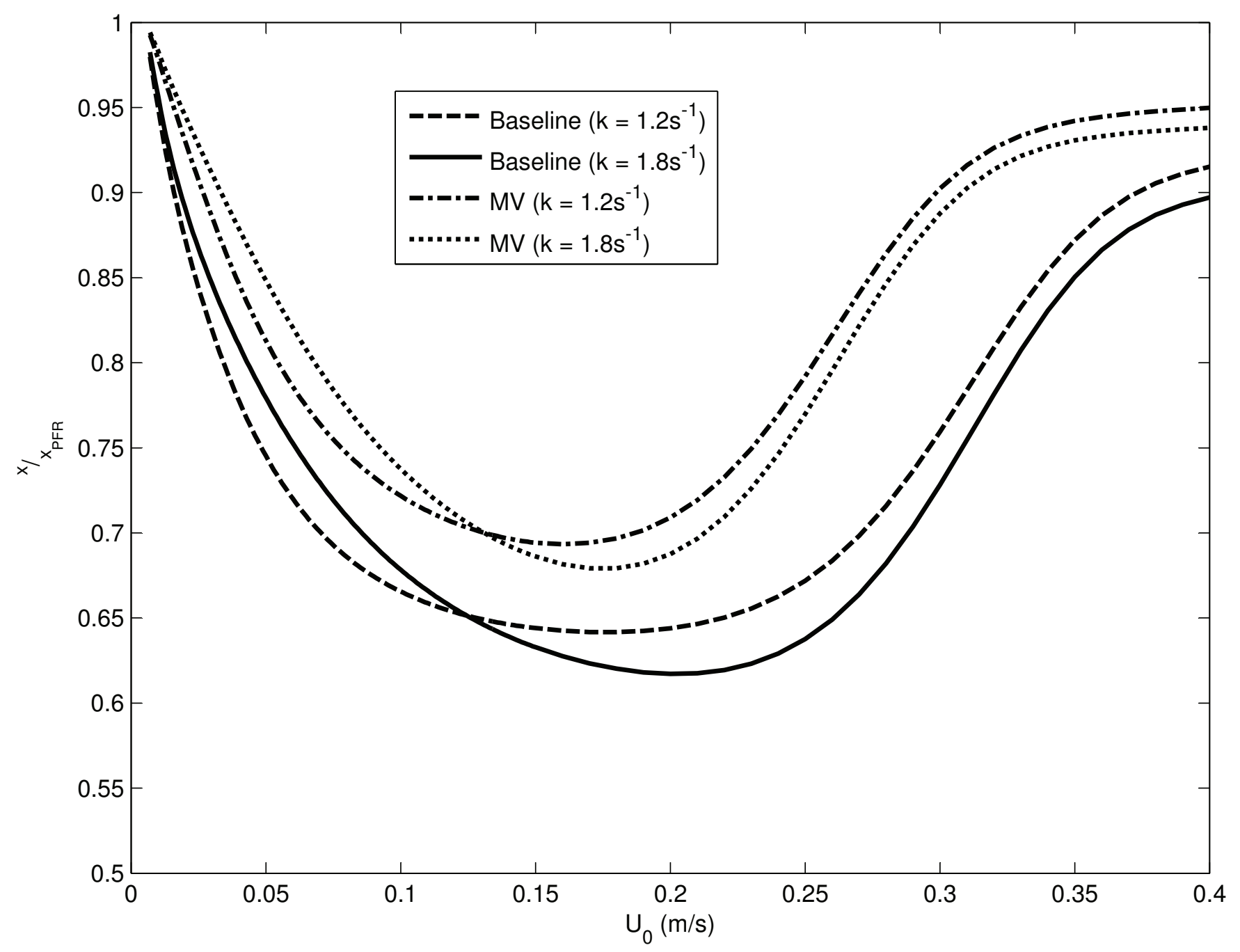

